\title{
Transit time flow measurement in composite arterial revascularisation
}

\author{
R. John L. Brereton \\ Cardiothoracic Surgical Unit, Royal North Shore Hospital, Sydney, Australia \\ Correspondence to: R. John L. Brereton, FRACS. Cardiothoracic Surgical Unit, Royal North Shore Hospital, Sydney, Australia. \\ Email: drjbadmin@bigpond.com.
}

Submitted Aug 22, 2018. Accepted for publication Sep 10, 2018.

doi: $10.21037 /$ acs.2018.09.03

View this article at: http://dx.doi.org/10.21037/acs.2018.09.03

\section{Introduction}

Neither off-pump nor on-pump coronary surgeries have good enough graft patency to forsake simple measures for improvement. In most other cardiac procedures, operative surveillance is undertaken, whether in valvular surgery or percutaneous intervention. Of the measures proposed for coronary surgery, transit-time flow measurement (TTFM) stands apart from duplex imaging, fluorescent angiography and contrast angiography as being readily implemented and non-invasive. TTFM results correlate with adverse cardiac events, graft failure, and mortality; sensible interpretative algorithms may prevent these dire consequences (1).

\section{Testing the probe}

The manufacturer recommends testing the probe prior to each use. Both the maximal Acoustic Coupling Index (ACI) and the zero-offset or baseline flow readout must be tested. Subtle damage to components and circuitry occurs from wear and electrostatic damage. Also, acoustic coupling may be improved by following the protocol. The probe is placed in saline within a plastic receptacle and agitated to remove bubbles. Immersed, the ACI or percentage of transmitted signal received should be greater than $90 \%$. In practice, this recommendation is punitively stringent-I find $80 \%$ more practical. The zero-offset should be minimal: the manufacturer does not provide a figure, but if greater than either $5 \mathrm{~mL} / \mathrm{min}$ or $5 \%$ of expected flow, then the probe should be unplugged and replugged to the alternative Q2 channel. If the zero-offset is still non-negligible, another probe should be used. Also, I recommend holding the probe immersed, swirling the saline clockwise and then counterclockwise to verify trace deflection in both directions (Figure 1A,B).

\section{Positioning the conduit}

The conduit should fit comfortably and snugly—but not compressed-within the transducer. Under-sizing the probe will limit flow by compression and some conduit will extrude, thereby underestimating flow. Oversizing the probe leads to overestimation of flow, particularly in the presence of high flow. With tapering arterial conduits, a position of good fit can usually be found; relative freedom from clips is helpful (Figure 2A). The conduit should be placed straight within the transducer: linear and not curved, perpendicular to the handle, parallel to the back wall. Curving and angulating the conduit can overestimate flow (e.g., by pushing downwards, Figure 2B), but generally underestimates flow (2).

\section{Free flow measurement}

Free flow should be assessed prior to suturing each proximal and distal anastomosis. This can be assessed visually, or rigorously by direct measurement and TTFM. With some forethought, blood loss is minimized. It is unwise to construct proximal inflows for composite grafts (I, T, K, $\pi$ ) before verifying available flow; it is difficult to interpret competitive flow if free flow has not been assessed. Harvest skeletonized conduits allow dilatation at systemic pressure after treatment with intraluminal verapamil (5 $\mathrm{mg}$ per $5 \mathrm{~mL}$ arterial blood) and topical papaverine. After making the proximal or distal arteriotomy and inspecting 

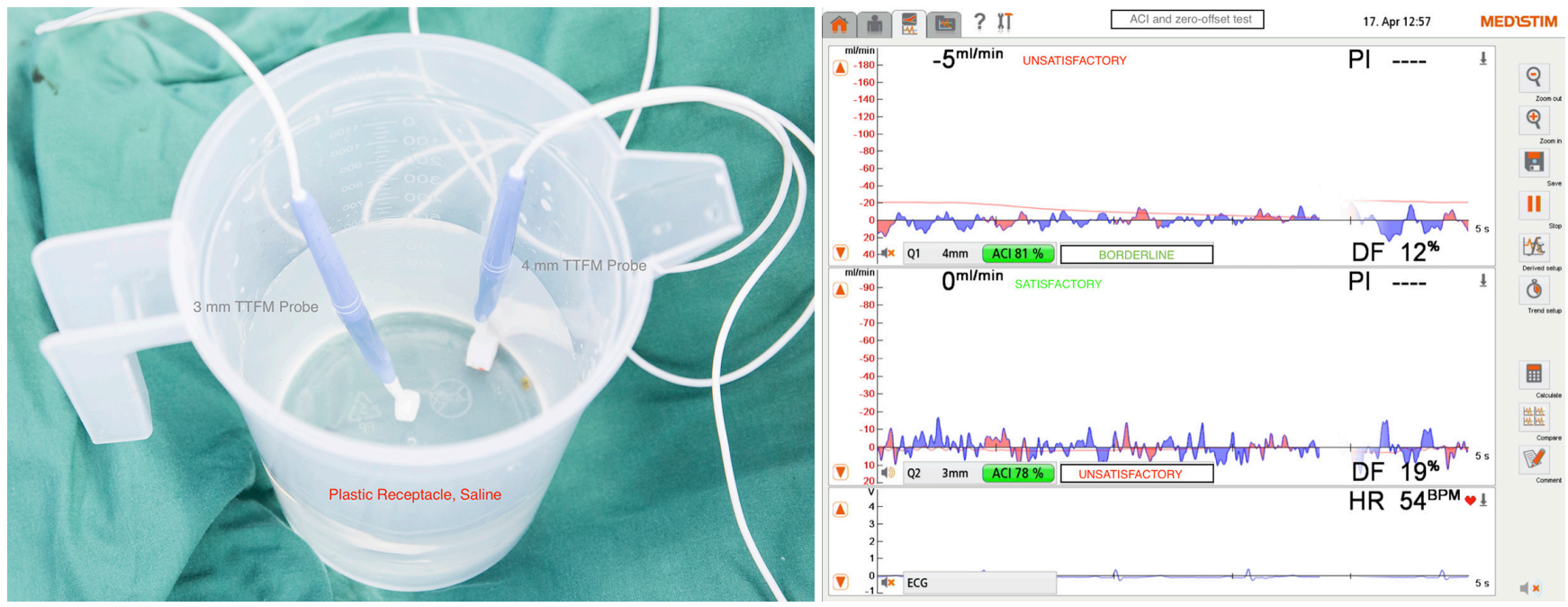

Figure 1 Probe testing. (A) TTFM probes immersed in saline in plastic container; (B) maximal acoustic coupling index and baseline zerooffset flows. TTFM, transit-time flow measurement.
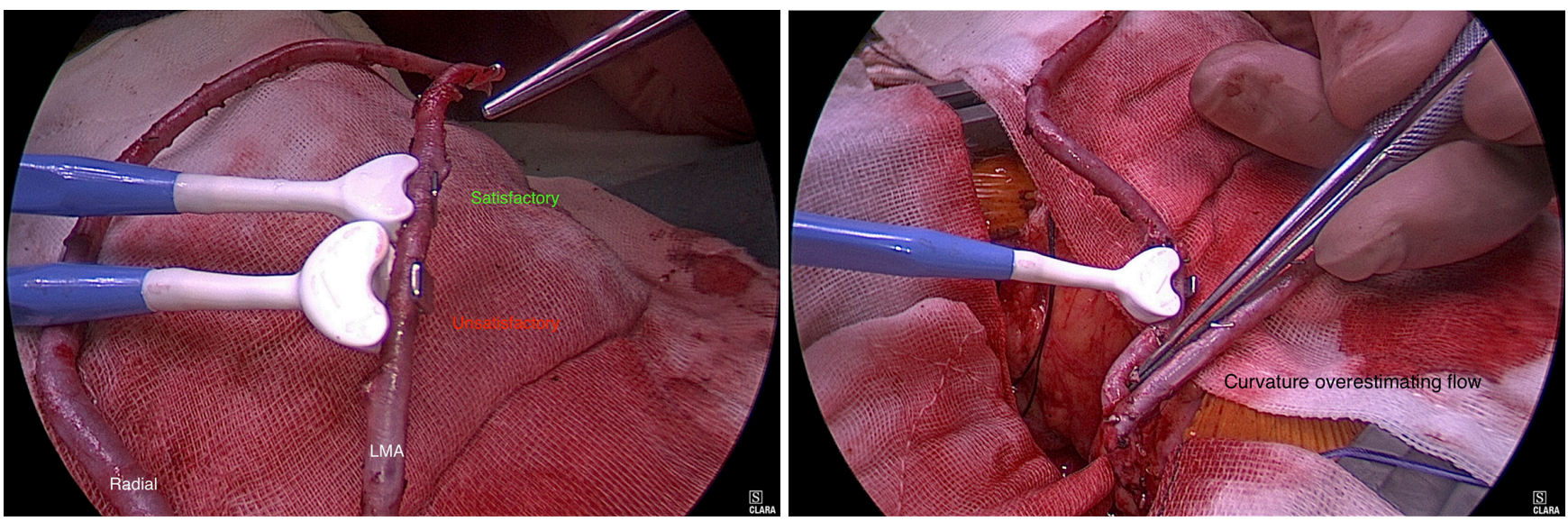

Figure 2 Conduit placement in probe. (A) Satisfactory vs. unsatisfactory fit and straight lie; (B) curved lie leading to flow overestimation.

for spasm, measure the time to bleed a measured volume, for example $10 \mathrm{~mL}$, into a syringe, the tip being occluded with bone wax. Calculate the flow from a hyperbolic flow curve. Comparison is made with the appearance and the concurrent TTFM reading (Figure $3 A, B, C$ ). Finding inadequate flow for the intended usage of course leads to revision of the operative plan.

\section{Anastomotic routine}

Surgeons should develop routines applicable to their particular techniques. The following routine allows a thorough evaluation of graft integrity and competitive flow in the anaortic, arterial, proximal first technique, with all distal technically side-to-side anastomoses:

(I) The stabilizer is positioned straddling the coronary, taking care to obstruct neither inflow or nor outflow;

(II) Small epicardiotomies are made to pass a silastic snare around the proximal vessel, fully encircling it, but not snugged (Figure 4A);

(III) The coronary arteriotomy is made and native anterograde flow is assessed as strong, impaired, or weak (Figure 4B); 

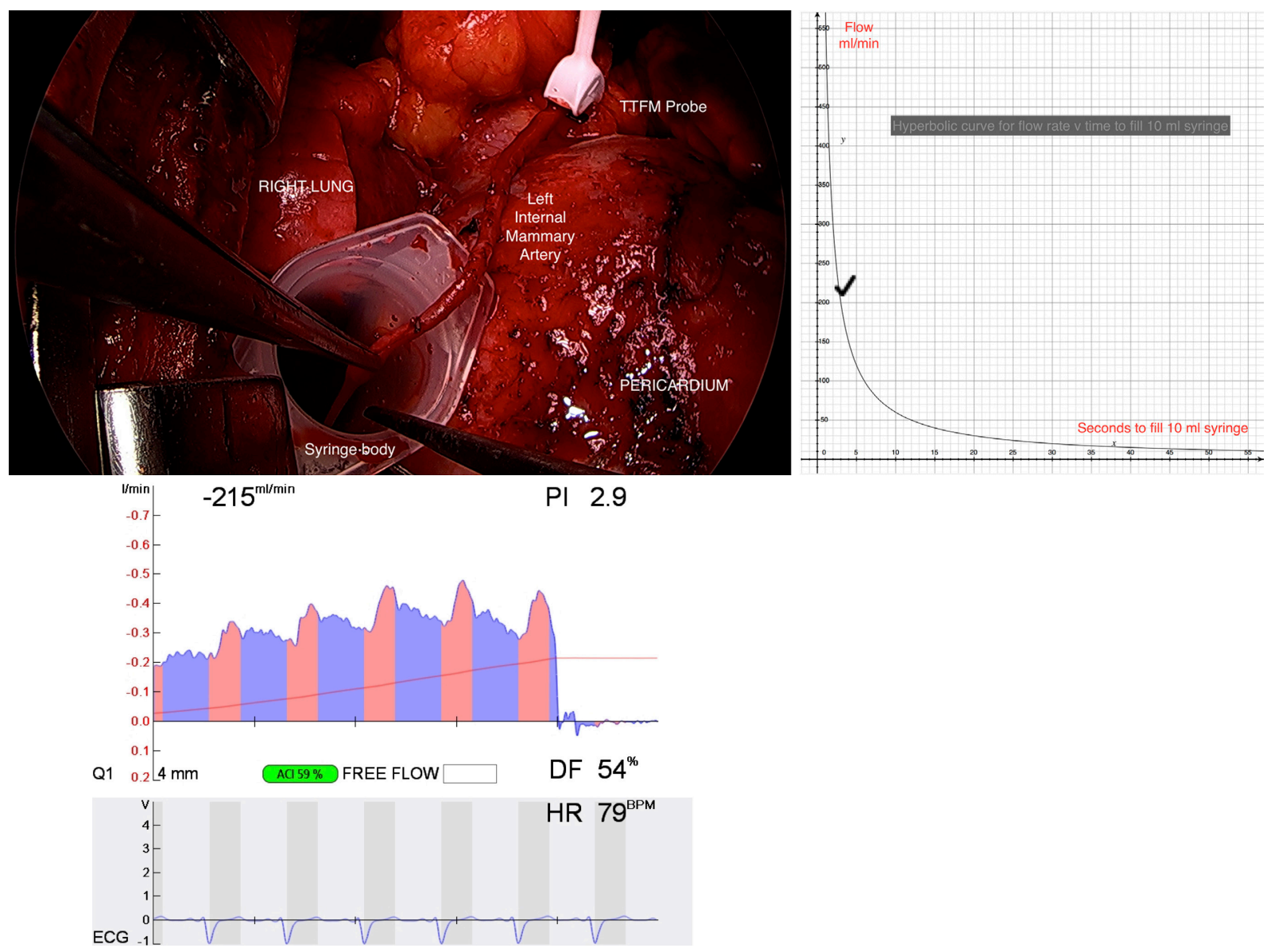

Figure 3 Estimation of free flow. (A) Free flow timed for a measured volume and assessed with TTFM; (B) hyperbola giving flow for a given time to fill to ten mis; (C) corresponding free flow trace on TTFM for mammary artery. TTFM, transit-time flow measurement.

(IV) The snare is snugged to assess retrograde collateral flow as strong or weak, oxygenated or de-oxygenated;

(V) A silastic shunt is passed proximally and distally;

(VI) The arterial conduit is incised, to allow a side-toside anastomosis. Free graft flow is again visualised and can be measured directly and with TTFM (Figure 4C);

(VII) The anastomosis is sewn, but before the knot is tied, the shunt is removed and the snare snugged, stopping native flow;

(VIII) The bulldog clamp is removed from the conduit and the suture taken up and tied with unrestricted conduit flow. The graft flow is measured with TTFM;

(IX) The snare is removed to allow through competitive native flow. In this manner, flow parameters are recorded without and then with competition
(Figure 4D). If satisfactory, proceed to the next anastomosis;

(X) After completion of all anastomoses and administration of protamine, remeasure those grafts that can be checked safely.

\section{Interpreting results}

Important threshold measurements have been established from clinical practice (3). As the trace appears, judge the graft in this order:

(I) A graft with a pulsatility index of greater than 5 should be regarded with great concern. This correlates unarguably with adverse post-operative events, poor patency and possibly mortality. It is vital to comprehend that despite assessing a graft in terms of flow, electrocardiograph and echo, a 

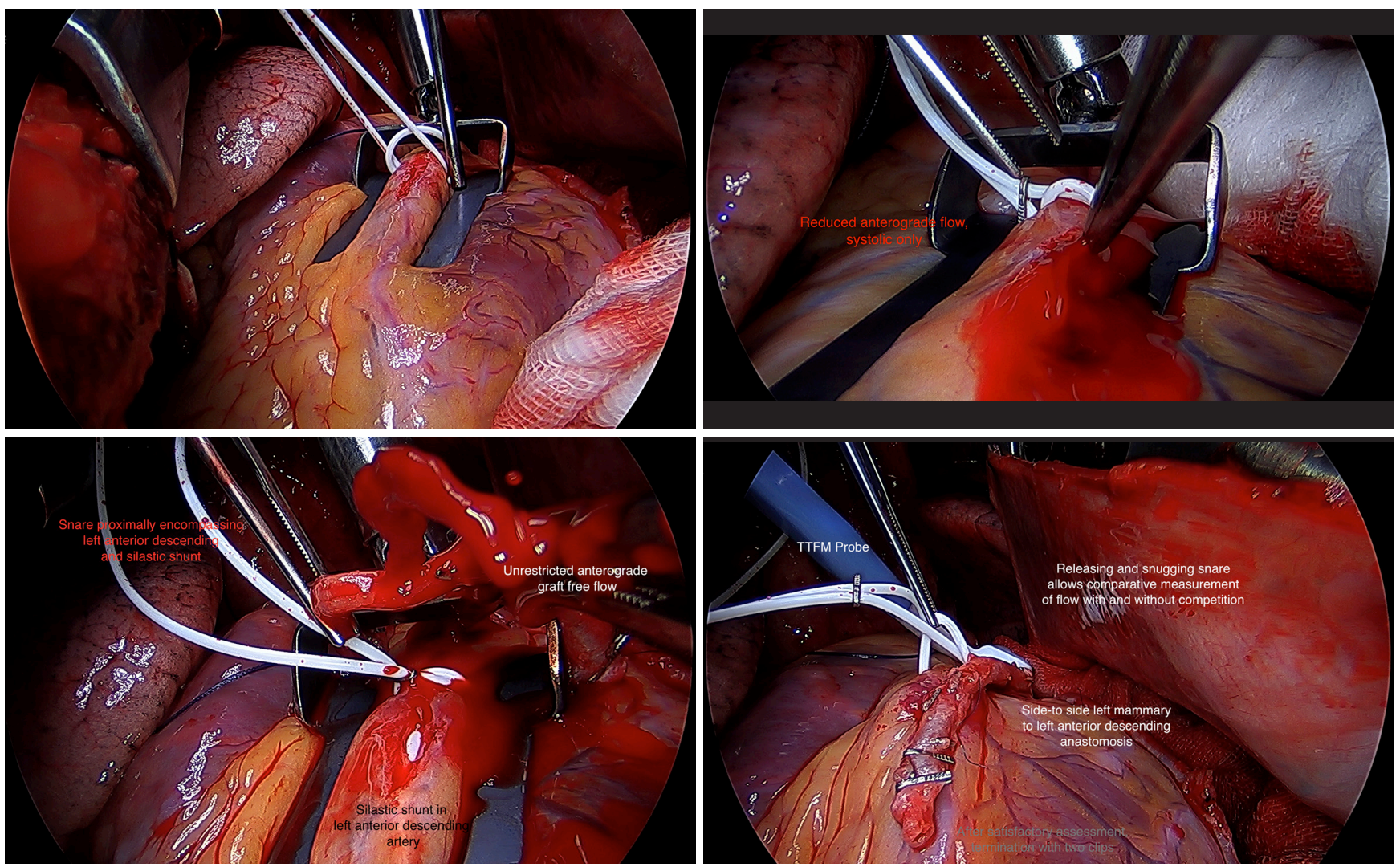

Figure 4 Anastomotic technique. (A) Coronary with loose proximal snare; (B) reduced anterograde flow at arteriotomy; (C) strong mammary artery free flow, shunt in left anterior descending artery; (D) snugging and releasing snare to allow comparison of graft flow with and without native competition.

patient with a graft wherein the pulsatility index (PI) is greater than 5 is at greater risk of adverse outcome (Kieser et al. 2010);

(II) A measured flow of less than $15 \mathrm{~mL}$ per minute correlates with poorer graft patency and adverse post-operative events. Although sometimes satisfactory, it is important to reconsider the function of this graft in terms of territory perfused (Kim, Kang and Lim 2005);

(III) A pulsatility index of less than 3 is desirable in grafts to the left ventricular territory, including the occluded posterior descending; that is grafts not running off into right ventricular territory. If greater than 3 , an explanation such as competitive flow should be sought, or patency is compromised (4);

(IV) A measured flow of greater than $30 \mathrm{~mL} / \mathrm{min}$ correlates with longer term graft patency;

(V) Diastolic flow should be greater than $50 \%$, probably $60 \%$ in "left-sided" grafts.

\section{Dealing with borderline results}

Interpretation of flow requires greater experience and clinical judgement than that of PI. As of yet, there is no algorithm to predict flow in a given situation. However, knowing total coronary flow, the surgeon should hypothesize a flow before measuring, considering the sizes of the conduit, the coronary and the territory perfused, in terms of the severity of the coronary stenosis. Individual clinical circumstances must be carefully understood by the surgeon. For example, if an acute left main occlusion is to be replaced by dual arterial grafts based on bilateral mammary inflows and bidirectional outflows, then the free flow must exceed and graft flow be consistent with total coronary blood flow of $250 \mathrm{~mL} / \mathrm{min}$, with an allowance to be made for the effects of acute ischaemia and intra-aortic balloon counter-pulsation. Conversely, a left mammary anastomosed 
to a diffusely diseased left anterior descending in the distal sixth may reveal a flow of only $15 \mathrm{~mL} / \mathrm{min}$, but still provide sufficient flow reserve to reverse exercise-induced apical systolic failure. There is a series of steps which must be undertaken before taking down the anastomosis:

(I) Is inflow disrupted? Check that the systemic blood pressure is satisfactory, within the levels of autoregulation, and examine the graft from origin to anastomosis for kinks or compression;

(II) Is the target correct? With reference to the coronary angiogram, is the correct vessel grafted in the correct position beyond disease?

(III) Is there flow restriction from adventitial bands, particularly at the inflow, or kinking at the heel, or buckling in the skip? This may be remedied without re-doing the anastomosis;

(IV) Is the flow consistent with the conduit and coronary free flow? Recheck both through the cut distal end beyond the side-to-side anastomosis, or at the next graft arteriotomy;

(V) If competitive flow is eliminated by snaring, does the PI reduce to satisfactory levels and flow improve?

(VI) Is flow consistent with the size of the distal coronary bed, was there strong retrograde flow, should the graft be perfusing proximally and distally from the anastomosis or only distally?

(VII) Is the anastomosis sound? Was every suture visualised at the intimal surface, could the outflow be narrowed, is the inflow kinked, could the other side of the conduit be picked up in the suture, were extra sutures placed, could the anastomosis be purse-stringed?

\section{Competitive flow}

If measured flow drops significantly with removal of the proximal native snare, competitive flow exists. Usually the PI rises concomitantly. This does not necessarily imply that the coronary stenosis is insignificant; it carries other implications. Competitive flow requires evaluation of both components: the native vessel and the conduit:

(I) Is the target correct? With reference to the coronary angiogram, is the correct vessel grafted in the correct position beyond disease? For example, mistakenly grafting a diagonal branch instead of the left anterior descending will show this effect;

(II) Is conduit flow limited by correctable bands, kinks, stenoses? Could the conduit be shortened to allow an anastomosis using a portion of greater radius and therefore markedly less resistance to flow? This is particularly relevant to tapering mammary arteries. Should the inflow be changed?

(III) Are there excessive demands on the inflow, for example, a small mammary, or the left mammary saphenous vein $\mathrm{T}$ graft (wherein flow in the terminal left mammary to a moderately stenotic left anterior descending characteristically suffers competitive flow)? Snare the other limb, retest, and consider alternative inflows.

\section{Conclusions}

The European Association of Cardiothoracic Surgery Guidelines on Myocardial Revascularisation recommend TTFM for confirming or excluding technical graft problems. In arterial revascularisation flow measurement provides the dual advantages of verifying graft function and quantitating competitive flow. As a teaching tool, TTFM is valuable in ensuring that anastomoses performed by trainees are sound. To surgeons pursuing the development of anaortic, minimally invasive and robotic surgery, the security proffered is both indisputable and indispensable. Although not without value when used occasionally in dire ischaemic circumstances, TTFM is of most value when used both routinely and with a strict routine. It appears that most surgeons committed to off-pump arterial revascularisation use TTFM and are beginning to adopt epicardial duplex scanning.

\section{Acknowledgements}

Transit Time Flow Equipment has been provided by the North Shore Cardiothoracic Research and Education Fund.

\section{Footnote}

Conflicts of Interest: The author has no conflicts of interest to declare.

\section{References}

1. Kieser TM, Rose S, Kowalewski R, et al. Transit-time flow predicts outcomes in coronary artery bypass graft patients: a series of 1000 consecutive arterial grafts. Eur J Cardiothorac Surg 2010;38:155-62.

2. Amin S, Werner RS, Madsen PL, et al. Intraoperative Bypass Graft Flow Measurement With Transit Time 
Flowmetry: A Clinical Assessment. Ann Thorac Surg 2018;106:532-8

3. Kieser TM. Graft quality verification in coronary artery bypass graft surgery: how, when and why? Curr Opin Cardiol 2017;32:722-36.

Cite this article as: Brereton RJ. Transit time flow measurement in composite arterial revascularisation. Ann Cardiothorac Surg 2018;7(5):710-715. doi: 10.21037/ acs.2018.09.03
4. Kim KB, Kang CH, Lim C. Prediction of graft flow impairment by intraoperative transit time flow measurement in off-pump coronary artery bypass using arterial grafts. Ann Thorac Surg 2005;80:594-8. 